\title{
SCULTURE ALTOMEDIEVALI LUNGO IL BASSO CORSO DELL'ADIGE (SECOLI VI-X)
}

PAOLO VEDOVETTO

UDC: 726.54.033.1(450.34)"05/o9" $27-526$

Original scientific paper

Manuscript received: 01. 02. 2016.

Revised manuscript accepted: 26. 03. 2016.

DOI: 10.1484/J.HAM.5.111361
P. Vedovetto

Dipartimento dei Beni Culturali Università degli Studi di Padova

Piazza Capitaniato 7 35139 Padova, Italia

The recent update of the catalogue of the early medieval churches in the Padua province allowed us to record more buildings built before the $10^{\text {th }}$ century, across the course of the Adige river between the towns of Montagnana (Padua) and Brondolo (Chioggia, Venice) (see Brogiolo in this volume). This paper deals with 17 fragments of liturgical furniture, some previously unpublished; for some churches these pieces offer the only surviving proof of their early medieval foundation. This work is a preliminary study of the Corpus of Early Medieval Sculpture of the dioceses of Padua and Chioggia, under investigation by the author.

Key words: Veneto, Adige, Montagnana, Chioggia, VI th century, early medieval churches, CARE, spolia, sculpture, liturgical furniture

Ad un'assenza quasi totale di fonti scritte relative all'organizzazione ecclesiastica nella parte meridionale della diocesi altomedievale di Padova, corrisponde l'estrema rarefazione delle attestazioni materiali pertinenti a chiese. Recenti indagini hanno favorito l'identificazione di nuove testimonianze architettoniche in alzato anteriori al X secolo, che si vanno a sommare ai repertori già noti in letteratura (Brogiolo in questo volume); tuttavia, l'esistenza di questi edifici è spesso indiziata solamente da resti di arredo liturgico e di decorazione architettonica, il più delle volte erratici oppure reimpiegati in contesti edilizi successivi. Si tratta complessivamente di diciassette manufatti, per un'area corrispondente a tredici circoscrizioni pievane (fig. 1).
Iniziando da ovest, un frammento di pluteo in marmo, finora inedito, si trova riadattato come acquasantiera nella chiesa del monastero francescano di Santa Maria delle Grazie (detta "Madonna di Fuori") presso Montagnana (Pd) ${ }^{1}$. Il reperto presenta uno schema decorativo a Korbodden, definito da un cerchio campito da una losanga, delineati da tre listelli lisci attraversati in diagonale da una matassa a due capi di tre vimini. Negli spazi di risulta tra il cerchio e la losanga compaiono foglie lanceolate profilate e curvate; il quadrato ospita, invece, un motivo a $\mathrm{S}$ affrontate. Lo schema, diffusissimo in Italia centrale, trova confronti puntuali anche in Italia settentrionale e di là delle Alpi tra la fine dell'VIII secolo e la metà del IX (fig. 2)². Se allo stato attuale

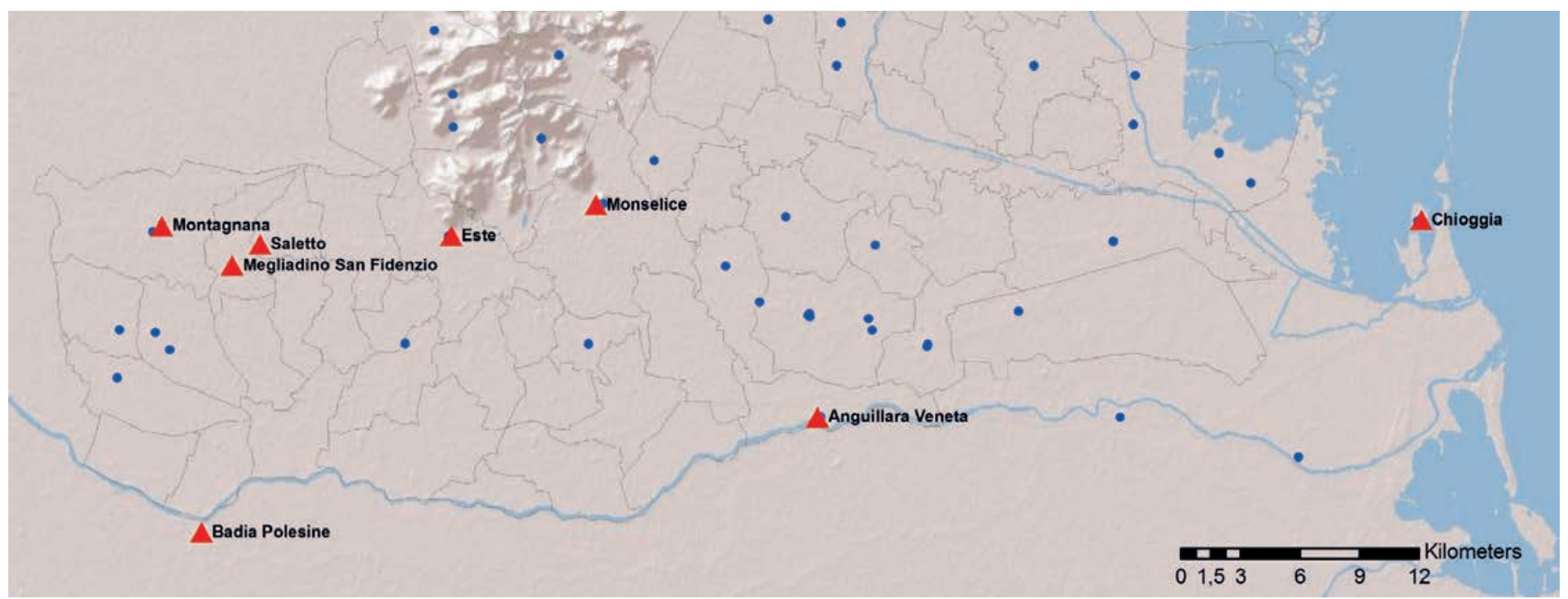

Fig. 1. Localizzazione dei luoghi di culto attestati prima del X secolo e delle sculture altomedievali oggetto di studio.

${ }^{1}$ L'attuale oratorio fu ricavato dalla cappella laterale nord della chiesa rinascimentale, demolita nel 1778 (alcune notizie sulla chiesa in A. CORRADIN, Chiesa e convento della Madonna di Fuori di Montagnana (1504-1778): notizie storiche, Montagnana, 1986). Il manufatto presenta le seguenti dimensioni: cm 32 (raggio); $\mathrm{cm} 12$ (spessore). Grazie alla parziale ricostruzione del motivo possiamo ipotizzare che il frammento appartenesse a un pluteo quadrato di ca. cm 70 di larghezza.

${ }^{2}$ Confronti a Venezia, in un frammento proveniente dalla base del campanile di San Marco (M. PILUTTI NAMER, Reimpiego e rilavorazione di materiali antichi nella Venezia medievale: alcuni esempi, in Antichità altoadriatiche, 74, 2012, p. 165, fig. 7); Murano (M. VECCHI, Sculture tardo-antiche e altomedievali di Murano, Roma, 1995, nn. 4-5); Torcello (R. POLACCO, Sculture paleocristiane e altomedievali di Torcello, Treviso, 1976 n. 66); Sirmione 


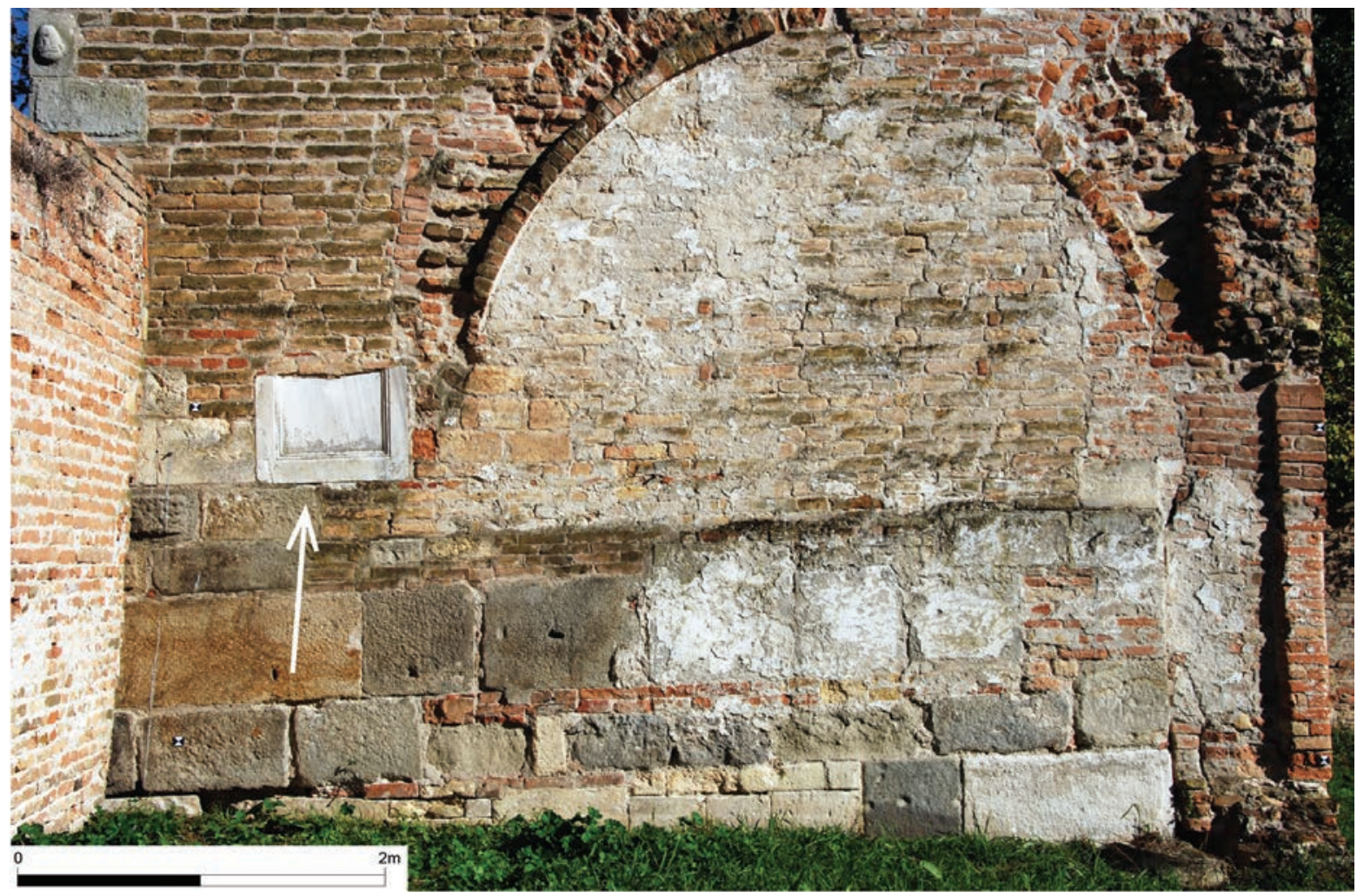

Fig. 3. Badia Polesine (Ro), Santa Maria della Vangadizza, parte basale del campanile con elementi di reimpiego.

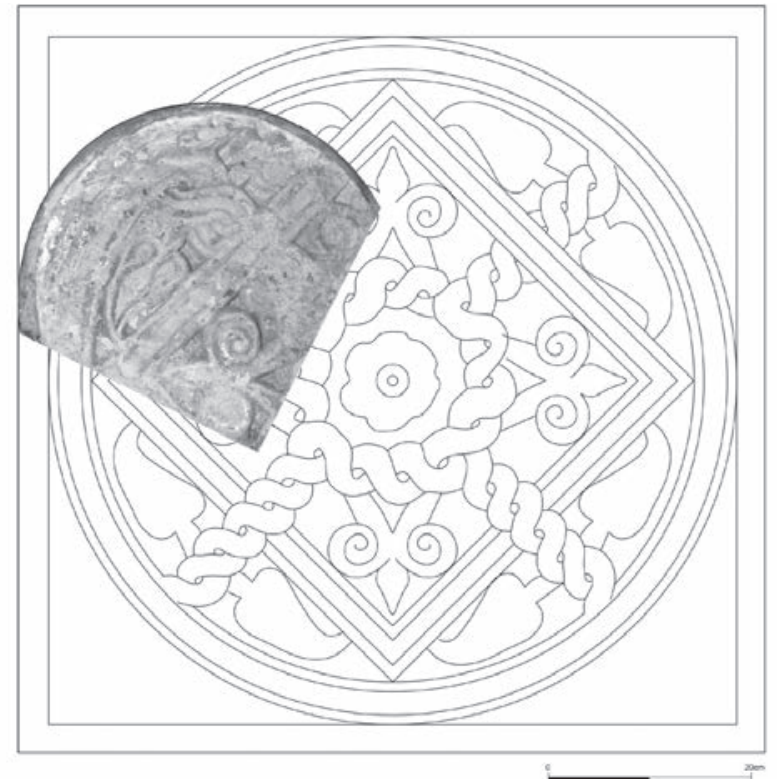

Fig. 2. Montagnana (Pd), Santa Maria delle Grazie, frammento di pluteo. è pressoché impossibile precisare la provenienza originaria del pezzo, possiamo tuttavia escludere la sua pertinenza all'arredo di Santa Maria delle Grazie, costruita solo nel $1504^{3}$, e ipotizzarne l'appartenenza ad una chiesa altomedievale dell'immediato circondario.

Più a sud, ai confini con il territorio veronese, un frammento di mensa di altare marmorea è reimpiegato nel lato meridionale del campanile della chiesa di Santa Maria della Vangadizza presso Badia Polesine $(\mathrm{Ro})^{4}$. La lastra, riconducibile a una tipologia comune in età tardoantica ${ }^{5}$, è caratterizzata da un bordo aggettante costituito da una fascia, un listello e un tondino, che incornicia un campo centrale liscio. Inglobato nella muratura in occasione dell'edificazione della torre campanaria per mano del magister Placent(invs) verso la fine del XI secolo, il manufatto apparteneva forse all'arredo liturgico del primitivo edificio di culto, precedente a quello fatto costruire da Almerico II agli inizi del X secolo (fig. 3$)^{6}$.

Un terzo frammento, oggi disperso ma di cui rimane una documentazione fotografica (fig. 4) ${ }^{7}$, fu scoperto nell'aprile del 1941 in via XX settembre presso Saletto, a est di Monta-

S. LUSUARDI SIENA, L'arredo architettonico e decorativo altomedievale delle chiese di Sirmione, in G. P. BROGIOLO, S. LUSUARDI SIENA, P. SESINO (a cura di), Ricerche su Sirmione longobarda, 1989, Firenze, p. 108; Vigo Lomaso (P. PORTA, Per il corpus della scultura altomedievale: la diocesi di Trento, in G. P. BROGIOLO et alii (a cura di), APSAT 10. Chiese trentine dalle origini al 1250, Volume 1, Mantova, 2013, pp. 50-51); Schänis (K. ROTH-RUBI, Die Flechtwerkskulptur Churrätiens-Müstair, Chur, Schänis, in Zeitschrift für schweizerische Archäologie und Kunstgeschichte, 67, 1/2, 2010, p. 21); Malles (N. RASMO 1981, Arte carolingia nell'Alto Adige, Bolzano, pp. 41-42).

3 L. WADDING, Annales Minorum, XV, 289, p. 334.

${ }_{4}^{4}$ Il manufatto è murato a un'altezza di circa $\mathrm{m}$ 1,90 da terra. Dimensioni: $\mathrm{cm} 63$ (larghezza max.) x 82. La base della torre campanaria reimpiega, oltre alla mensa, numerosi blocchi in trachite e manufatti romani. Sono anche riutilizzati come elementi decorativi tre conci con protomi umane quasi a pieno rilievo (têtes coupées), una nell'angolata N/W, due in quella di S/W, databili tra X e XI secolo.

5 Per una panoramica della tipologia si vedano i contributi contenuti in "HAM", n. 11 (2005).

${ }^{6}$ La prima attestazione risale al 953, quando Almerico II, con la moglie Franca, beneficiò la scola sacerdotum della chiesa della Beata Vergine Maria Madre di Dio, che essi stessi avevano fatto edificare e possedevano (J.B. MITTARELLI, D.A. COSTADONI, Annales camaldulenses ordinis Sancti Benedicti, I, app. col. 48). ${ }^{7}$ La prima notizia sul manufatto si trova in A. CALLEGARI, Saletto di Montagnana, in Notizie degli scavi di antichità, Roma, 1948, fascicolo I, VII, p. 11. Si veda, inoltre, A. GIACOMELLI, Notizie e ricerche per la storia di Montagnana e del suo territorio dalle origini al mille di Cristo, Montagnana, 1976, pp. 292294, fig. 8. Ai tempi dell'autore il manufatto era conservato presso il Museo Nazionale Atestino con il numero di inventario 18348. Dimensioni riportate: cm 74 (altezza) x 42 (larghezza) x 21 (spessore). 


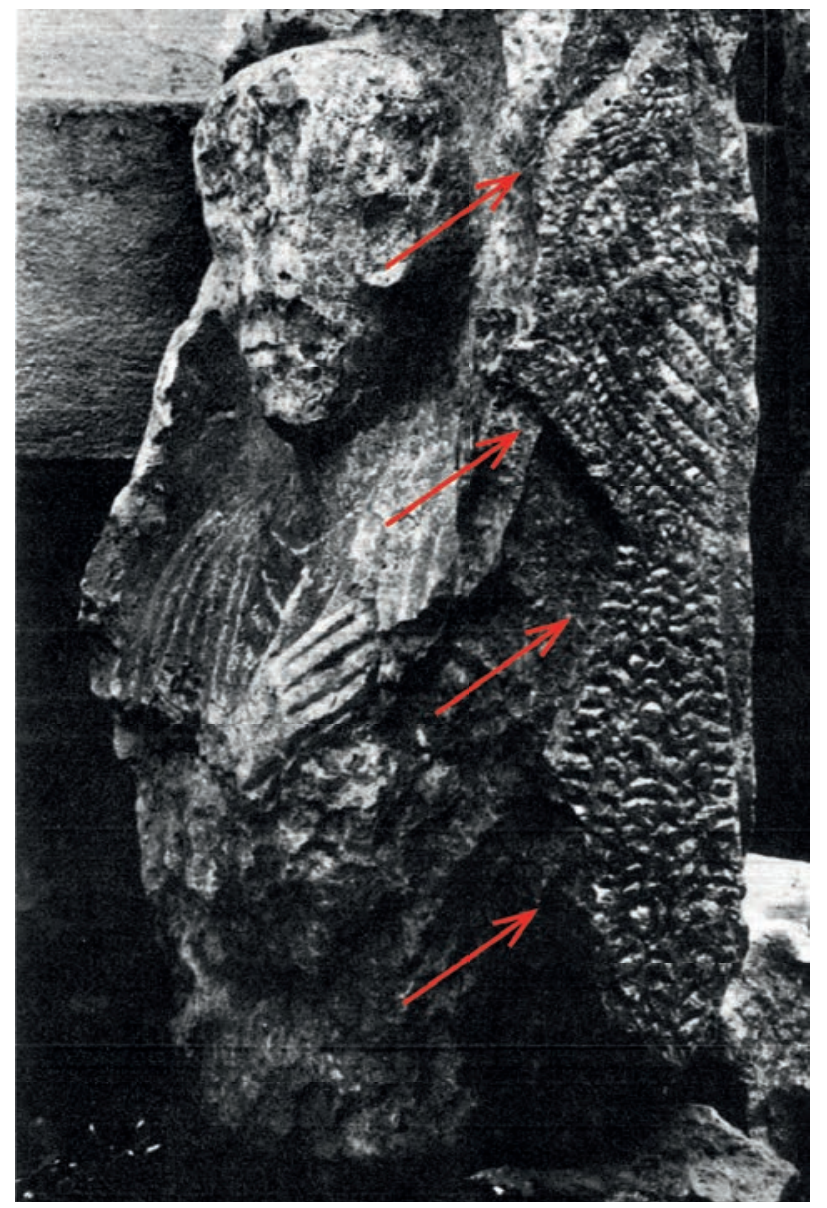

Fig. 4. Saletto di Montagnana (Pd), edicola funeraria con traccia di reimpiego (da Giacomelli 1976, p. 293).

gnana, da cui dista circa $5 \mathrm{~km}$ : si tratta di una stele funeraria in trachite di età romana (I sec. d.C.), riscolpita su un fianco con la figura stilizzata di un pavone raffigurato di profilo. Il soggetto potrebbe essere compatibile con una cronologia altomedievale difficilmente precisabile, in assenza di dati circa l'identità dell'edificio di provenienza ${ }^{8}$.
Più significative sono le testimonianze relative alla chiesa di San Fidenzio a Megliadino (Pd); qui nel 1906, in occasione della demolizione dell'edifico romanico, furono recuperati un frammento di lettorino di ambone monolitico, murato probabilmente nel perimetrale esterno dell'edificio ${ }^{9}$, e un sarcofago con l'iscrizione funeraria di Iuhannes presbiter, rinvenuto sotto il pavimento della cappella a sud dell'abside, assegnata tradizionalmente al X-XI secolo ${ }^{10}$ ma che andrebbe riportata all'VIII su basi paleografiche" (fig. 5).

La lastra di ambone, assai nota ${ }^{12}$, presenta una frattura regolare nella parte inferiore, forse funzionale al reimpiego. La parte superiore è coronata da un motivo a onde correnti con caulicoli forati, distribuite disordinatamente ma con l'intento di rendere una disposizione speculare rispetto all'asse centrale della lastra. Una croce latina divide lo spazio centrale in quattro comparti rettangolari, ciascuno scolpito al centro con un fiore a otto petali con bottone forato, incluso in un clipeo perlato/cordonato tra due listelli lisci, dal quale dipartono quattro gigli a foglie profilate. L'asse longitudinale è decorato con un motivo a tralcio vegetale a due vimini con foglie contrapposte, analogo a quello del bordo destro; l'asse orizzontale è percorso da un girale di foglie a goccia, profilate, desinenti alternativamente in una testa di serpente e una foglia profilata a tre lobi, che nascono ora dal basso ora dall'alto. Il bordo sinistro, infine, presenta un tralcio a cornucopia con foglie pendule. Puntuali paralleli con alcune sculture di area friulana ${ }^{13}$ hanno permesso di assegnare la lastra a una maestranza attiva a Cividale nel complesso regio di San Giovanni e Santa Maria in Valle intorno al terzo quarto dell'VIII secolo. Rimane a questo punto da chiarirne la sistemazione originaria, in mancanza di dati sulla chiesa altomedievale. Stabilita l'impossibilità di riferirlo ai più tardi interventi di rinnovamento del luogo di culto, eseguiti nel 970 per opera del vescovo Gauslino in occasione del trasferimento del corpo di San Fidenzio da Polverara $(\mathrm{Pd})^{14}$, non sembrerebbe improbabile una sua appartenenza all'arredo liturgico del già esistente oratorio di San Tommaso, in una fase in cui dovette assumere un'importanza tale da richie-

\footnotetext{
${ }_{8}^{8}$ La destinazione originaria di questo singolare manufatto rimane incerta; non possiamo escludere che facesse parte di un rilievo decorativo articolato (cornice?); si nota, infatti, la traccia di un profilo a $\mathrm{L}$ sul lato destro, funzionale forse all'alloggiamento di una lastra laterale.

9 Tre indizi farebbero pensare a un reimpiego esterno del pezzo: alcune tracce di intonaco lungo i margini, resti di dilavamento superficiale (A. RUTA SERAFINI, Scheda 15, in Restituzioni. Tesori d'arte restaurati, Venezia, 2008, p. 155) e la testimonianza dello storico Andrea Gloria che nel 1862 vide "infisso nel muro esterno un coperchio sepolcrale lavorato a rilievi anteriore al mille"(A. GLORIA, Il territorio padovano illustrato, v. 2, 1862, Padova, p. 343).

${ }^{10}$ Si veda M. SANNAZARO, Padova. Il territorio padovano, in A. CASTAGNETTI (a cura di), Il Veneto nel Medioevo, Verona, 1989, p. 250. Per una prima notizia del rinvenimento cfr. A. PROSDOCIMI, Regione X (Venetia). Nuove scoperte di antichità nel territorio Atestino. Migliadino S. Fidenzio, in Notizie degli scavi di antichità, XII, 1906, fascicolo 12, I, p. 420. Il sarcofago, oggi collocato all'interno della chiesa, presenta su uno dei lati lunghi la seguente iscrizione: "((Crux)) In n(omine) d(om)i(n)i Iesu Christi. Ego Iuhannes dixi pre(sbiter)/in privato". Dimensioni: cm 198 x 66 x 92 (spessore).

"Si osservi la A con traversa spezzata e la G costituita da una C caudata, lettere che compaiono con frequenza all'interno delle iscrizioni di livello medio basso dell'Italia settentrionale, comprese in un arco cronologico che tra l'inizio dell'VIII e il IX secolo, da Flavia De Rubeis definite come appartenenti alle maiuscole di tipo "capitale longobarda" (F. DE RUBEIS, Introduzione paleografica, in F. DE RUBEIS (a cura di), Inscriptiones medii aevi Italiae (saec. VIXII). Veneto - Belluno, Treviso, Vicenza, Spoleto, 2011, pp. 7-15.

${ }^{12}$ Si veda anche L. NAZZI, Amboni nell'area altoadriatica, Pasian di Prato (Ud), 2009, pp. 261-265 con ulteriore bibliografia. L'ambone fu pubblicato la prima volta nell'anno del suo rinvenimento (A. PROSDOCIMI, Regione X (Venetia)..., pp. 420-421) e dal 1907 è conservato presso il Museo Nazionale Atestino (I.G. 1859). Dimensioni: $\mathrm{cm} 87$ x 84,5 x 13 (spessore); altezza ricostruibile cm 110. Nella faccia superiore sono presenti due fori di trapano funzionali forse all'alloggiamento di un leggio ligneo (larghezza $\mathrm{cm}$ 1). Sulle facce laterali si riscontrano due incassi rettangolari, larghi $\mathrm{cm} \mathrm{4,5}$ e profondi cm 3,4, per la messa in opera di bassi plutei. Il tergo e lo spessore superiore sono lavorati a gradina.

${ }^{13}$ Il parallelo più puntuale proviene dalla pieve di San Martino a Rive d'Arcano (Ud) (S. LUSUARDI SIENA, L'arredo liturgico altomedievale, in S. LUSUARDI SIENA (a cura di), San Martino a Rive d'Arcano. Archeologia e storia di una pieve friulana, Udine, 1997, pp. 175-177); il motivo del tralcio scolpito nell'asse orizzontale della croce trova, invece, un riscontro molto puntuale nella vasca battesimale della pieve di San Marco a Rigoli (Pi), dove un fregio identico corre sul bordo superiore (A. M. DUCCI, La vasca battesimale di Rigoli: tra stile e tipologia, in Arte Medievale, v. 2, 1995, pp. 27-39).

${ }^{14}$ Stando alla leggenda dell'invenzione, il corpo santo fu poi trasportato lungo il fiume Vigenzone attraverso i centri di Bovolenta, Pernumia, Este, fino a Megliadino, ai confini meridionali della diocesi di Padova con quella di Verona (Proprium Sanctorum Ecclesiae Patavinae, pp. 56-63, Padova, 1933). Sulla figura del santo, con tutte le problematiche legate alla sua identificazione, si veda I. DANIELE, ad vocem San Fidenzio, in Bibliotheca Sanctorum V, Roma 1964, coll. 675-678.
} 

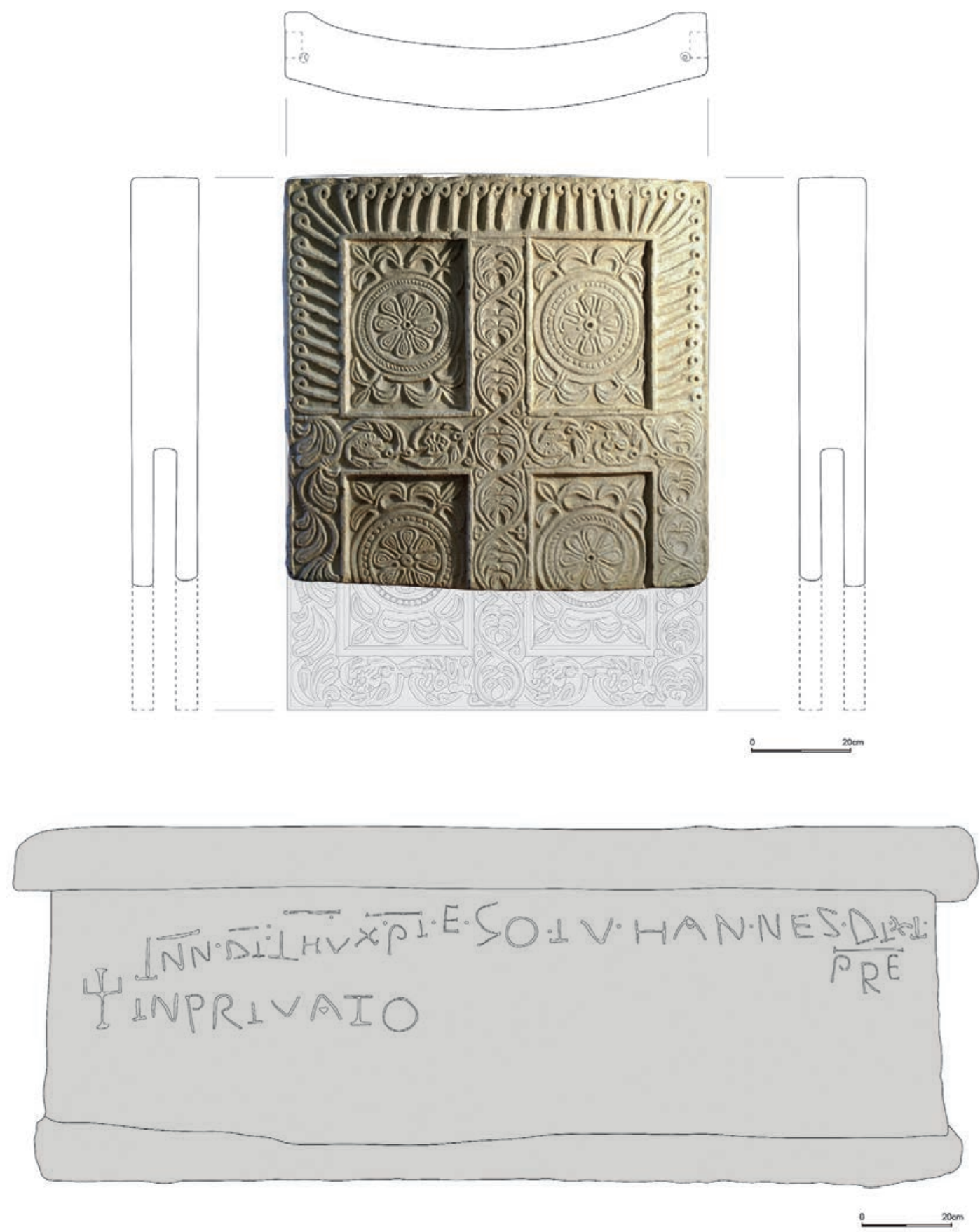

Fig. 5. Este (Pd), Museo Nazionale Atestino, lettorino d'ambone (ricostruzione), "su concessione del Ministero dei beni e delle attività culturali e del turismo"; riproduzione vietata. Megliadino, San Fidenzio, sarcofago del presbitero Giovanni (il rilievo epigrafico è stato eseguito con la collaborazione di Danilo Vitelli, sulla base dell'elaborazione delle immagini mediante il metodo RTI).

dere un arredo liturgico di alto pregio ${ }^{15}$; la lastra potrebbe essere stata poi reimpiegata nel perimetrale esterno della chiesa proprio in occasione dei lavori di rifacimento di fine $\mathrm{X}$ secolo $^{16}$. All'impianto altomedievale dell'edificio potremmo riferire anche un frammento di fusto in marmo cipollino conservato nella cripta ${ }^{17}$.
Vicini cronologicamente al pezzo di Megliadino sono due reperti provenienti da Este (Pd): un frammento di lastra con una treccia a tre nastri di tre vimini, incorniciata da due listelli e una fascia ad astragali ${ }^{18}$ e un frammento di capitello corinzio a foglie lisce ${ }^{19}$, murati rispettivamente nel perimetrale est e nord del campanile della chiesa di San Martino

\footnotetext{
${ }^{15}$ Un documento del 978 fornisce un riscontro documentario all'epigrafe murata nel 1685 all'interno della chiesa, dove si accenna all'esistenza di un oratorio campestre dedicato a San Tommaso apostolo, poi ingrandito, titolato a San Fidenzio e trasformato in collegiata (B. LANFRANCHI STRINA, Un documento inedito del 978 su S. Fidenzio di Megliadino, in Bollettino del Museo Civico di Padova, Padova, a. 62, n. 1-2, 1979, pp. 139-141).

${ }^{16}$ L'ipotesi avanzata da Silvia Lusuardi Siena di una provenienza dalla chiesa di Santa Maria "dell'Anconese" presso Megliadino San Vitale non è supportata dalle fonti documentarie, che la menzionano indirettamente solo dal 1145 e mai come pieve, cfr. S. LUSUARDI SIENA, Scheda n. 28o, in C. BERTELLI et alii (a cura di), Il futuro dei Longobardi. L'Italia e la costruzione dell'Europa di Carlo Magno, catalogo della mostra (Brescia 18 giugno-19 novembre 200o), Milano, 2000, p. 269.

${ }^{17}$ Dimensioni: altezza cm 176; diametro cm 32.

${ }^{18}$ Dimensioni: $\mathrm{cm} 51$ x 16,5.

${ }^{19}$ Dimensioni: cm 51 x 35 .
} 

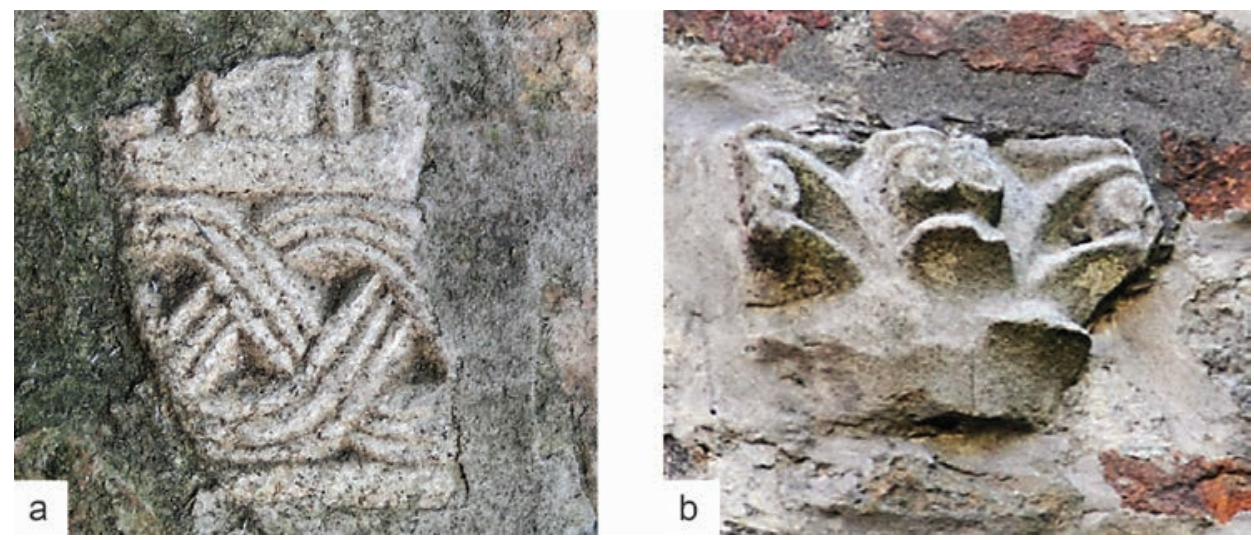

Fig. 6. Este (Pd), San Martino, campanile, frammenti.
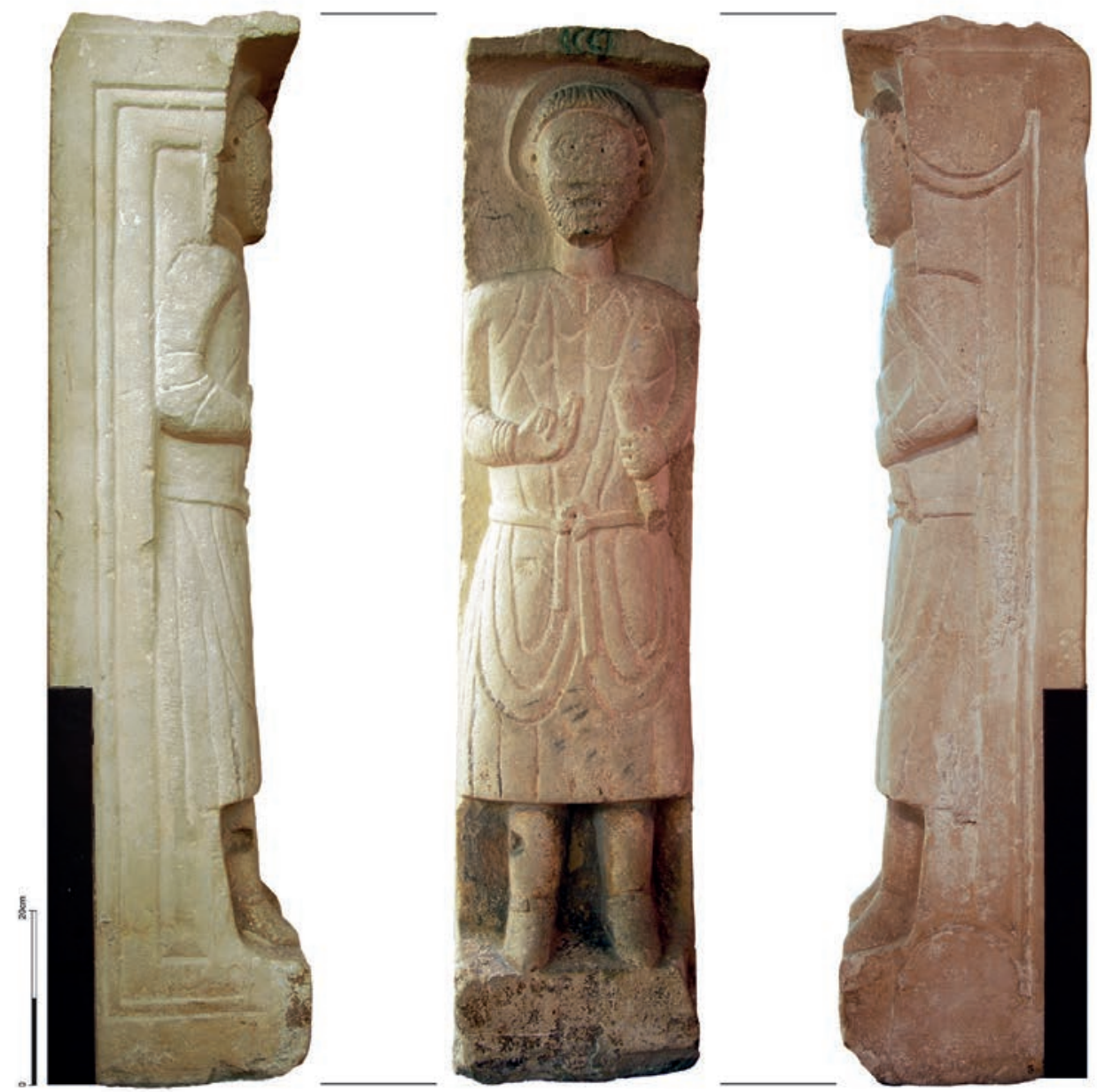

Fig. 7. Este (Pd), Museo Nazionale Atestino, pilastrino riscolpito, "su concessione del Ministero dei beni e delle attività culturali e del turismo"; riproduzione vietata.

(fig. 6). I manufatti potrebbero essere stati reimpiegati in occasione di alcuni lavori di rifacimento eseguiti nel 1293, testimoniati da un'epigrafe murata nel perimetrale nord della torre campanaria; dovrà essere quindi valutata la loro pertinenza ad un edificio precedente, per il quale costituiscono un terminus ante quem ${ }^{20}$.
Nel Museo Nazionale Atestino si conservano un pilastrino, riscolpito su un lato (fig. 7), e un fusto figurato (fig. 8), entrambi di provenienza ignota ma piuttosto omogenei per stile e tipo di lavorazione $e^{21}$.

Il pilastrino, pertinente a una recinzione presbiteriale $^{22}$ (lo si desume dalla presenza di un incasso laterale per l'inserimento di un pluteo, largo circa $\mathrm{cm}$ 8), ha un lato incorniciato da una modanatura composta da un listello a dente e una fascia con piano obliquo digradante. Sul lato opposto il campo rettangolare, incorniciato da una listello a dente, ha i lati brevi decussati, motivo che ricorre con frequenza su pilastrini tra VeVII secolo. Una faccia laterale appare riscolpita a altorilievo con la figura intera frontale di un giovane santo. Il capo aureolato è coperto da una capigliatura ondulata, definita da profondi solchi subparalleli e fori di trapano alle estremità, caratteristiche che troviamo riprodotte nella barba. Il volto ovale ha la bocca distinta da un netto solco orizzontale con forature di trapano successive che vanno a definire i denti; gli occhi sono tracciati a compasso, con foro centrale. La mano sinistra impugna un oggetto, frammentato alle estremità (lancia?) mentre la destra è in atteggiamento benedicente. L'uomo indossa una veste militare all'antica, con la lorica a squame e la cotta a strisce, chiusa in vita da una sottile cintura annodata al centro. In assenza di attributi iconografici specifici, si rivela al momento difficile l'identificazione del santo che possiamo genericamente far rientrare nella categoria dei martiri militari.

Il secondo esemplare in esame, un fusto in trachite probabilmente destinato al sostegno di una vasca, è scolpito su due lati con figure intere ad altorilievo le quali si inseriscono nel supporto per lo sviluppo dell'intera altezza ${ }^{23}$ : da un lato, Adamo ed Eva, in posizione rigidamente frontale, ai lati dell'albero intorno al cui tronco si attorciglia il serpente; dall'altro una non meglio definibile figura maschile indossa una lunga tunica solcata da pieghe, stretta in vita da una larga cintura con fibbia centrale e coperta da un mantello. La mano destra stringe un fiore a otto petali con bottone centrale mentre la sinistra tiene una mela. Quest'ultima figura appare strettamente imparentata con il santo militare

\footnotetext{
${ }^{20}$ La prima menzione della chiesa di San Martino di Este compare in un atto di donazione del 1026, oggi perduto (S. ORSATO, Historia di Padova, Padova, 1678, p. 228)

${ }^{21}$ I manufatti furono pubblicati per la prima (e unica) volta nella guida al museo di Adolfo Callegari del 1937, che si limitò a darne una sommaria descrizione, datandoli al XII secolo (A. CALLEGARI, Il Museo nazionale atestino in Este, Roma, 1937, p. 6).

${ }^{22}$ I.G. 1865; dimensioni: $\mathrm{cm} \mathrm{24,6}$ x 103,25 x 24,20 (spessore).

${ }_{23}$ I.G. 1866; dimensioni: cm 91 (altezza max), 29 (diametro superiore).
} 

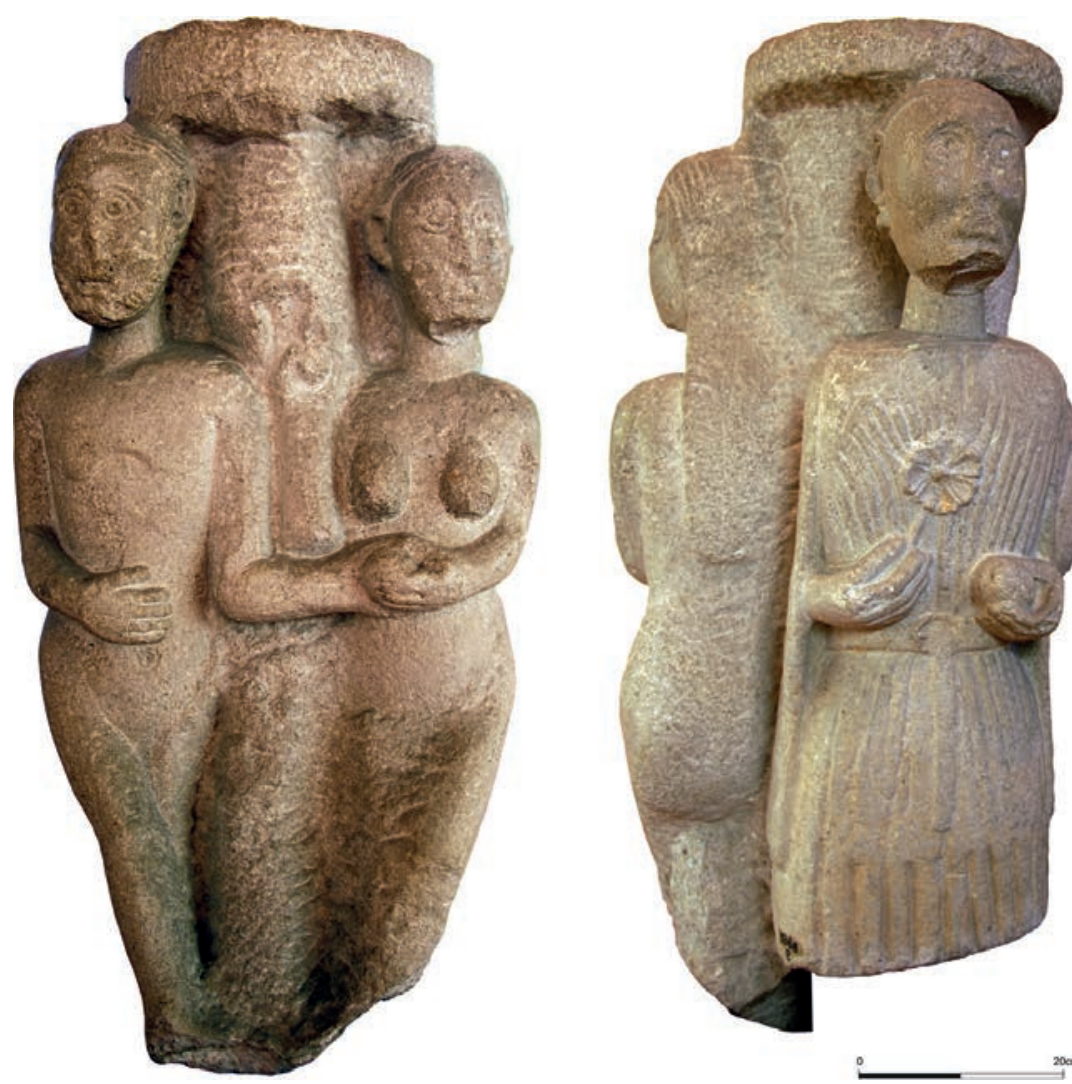

Fig. 8. Este (Pd), Museo Nazionale Atestino, fusto figurato, "su concessione del Ministero dei beni e delle attività culturali e del turismo"; riproduzione vietata.
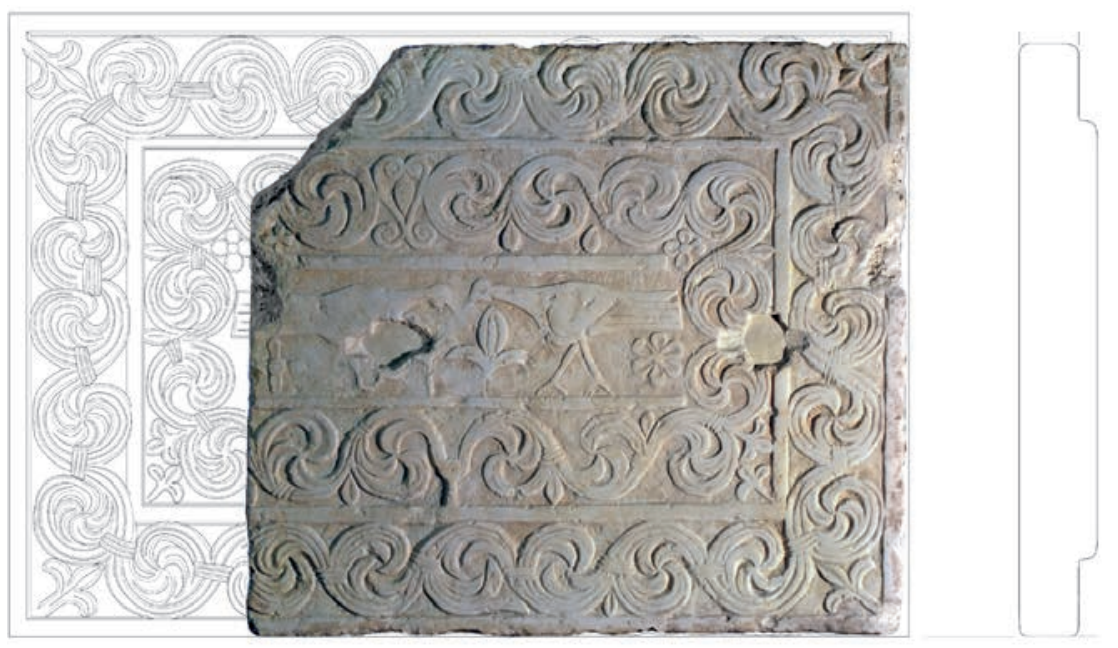

Fig. 9. Este (Pd), Museo Nazionale Atestino, pluteo da Anguillara Veneta (ricostruzione), "su concessione del Ministero dei beni e delle attività culturali e del turismo"; riproduzione vietata. (di cui replica l'attacco del collo, la postura, il grafismo delle vesti e la resa degli occhi) tanto da far pensare che entrambi i manufatti siano appartenuti allo stesso monumento. A fronte di problemi interpretativi e cronologici che devono necessariamente rimanere aperti, credo si possa con cautela avanzare l'ipotesi di una loro provenienza dalla vicina chiesa di Santa Tecla; in particolare il fusto figurato ben si accorderebbe con la colonna sormontata da un capitello e da una vasca battesimale vista dal vescovo Barozzi nel 1489 al centro del battistero romanico dedicato a San Giovanni Battista (v. Brogiolo in questo volume).

Il museo atestino conserva, inoltre, un pluteo frammentario proveniente dalla vecchia parrocchiale di Sant'Andrea apostolo di Anguillara Veneta $(\mathrm{Pd})^{24}$, ricostruita intorno al 1700 a nord dell'Adige, a circa $600 \mathrm{~m}$ a sud est dalla chiesa attuale, eretta tra il 1900 e il 1906 (fig. 9) ${ }^{25}$; non è dato conoscere in quale chiesa fosse originariamente, ma sappiamo che già nel 954 doveva esistere in questi luoghi una cappella dedicata probabilmente a Santa Maria ${ }^{26}$. La lastra è racchiusa da un listello piatto e presenta una cornice a tralcio di tre vimini con girali di foglie a "pale d'elica" con un motivo a gigli negli angoli. Un altro listello delimita una seconda cornice, simile nelle dimensioni a quella esterna dove si ripete lo stesso tralcio ma ridotto nelle proporzioni, caratterizzato da alcuni inserti fitomorfi (fiori a quattro petali, foglie a goccia) disposti in maniera casuale. Il registro centrale, delimitato da due listelli orizzontali, è scolpito con due pavoni disposti simmetricamente e con gigli e fiori schematici negli spazi di risulta. Il tralcio a "pale d'elica" privo di bottone centrale è piuttosto singolare e non è stato possibile individuare confronti esatti. Ciò che emerge è la totale assenza di simmetria e lo squilibrio compositivo che, assieme ad alcune imprecisioni esecutive ${ }^{27}$, sembrerebbero essere l'esito della libera interpretazione del lapicida di un cartone standardizzato.

Passando al centro pievano di Monselice (Pd), il più antico attestato dalle fonti scrit$\mathrm{te}^{28}$, le sculture conservate sono assai esigue in confronto al numero consistente di chiese altomedievali esistenti (v. Brogiolo in questo

${ }^{24}$ I.G. 1858. Dimensioni: cm 91,70 x 83,20 x 10,5 (spessore max.); Dimensioni originali ricostruibili: cm 126x 87,20. Il riutilizzo del pezzo come lastra di copertura tombale sembra essere confermato da molteplici indizi: la riduzione dello spessore del recto, abbassato di ca. $\mathrm{cm} 3$ e per una larghezza di $\mathrm{cm} 8$ lungo tutto il perimetro; i due fori passanti realizzati simmetricamente rispetto al centro della lastra, già ridotta dalle dimensioni originarie, forse riferibili a perni per il sollevamento; il degrado superficiale da calpestio (cfr. G. VALENZANO 2008, Scheda 14, in Restituzioni. Tesori d'arte restaurati, Venezia, 2008, pp. 146-151.

${ }^{25}$ L'antica chiesa è rappresentata nel catasto napoleonico del 1809 e in alcune mappe del XVIII secolo in corrispondenza dell'attuale via Canareggio (loc. Piazza Vecchia). La chiesa quattrocentesca era ad aula unica ( 12 x 5,2) conclusa da un'abside a est (Visit. Barozzi 1489).

${ }^{26}$ Tra i confini dei beni della curtis di Bagnoli (oggi Bagnoli di Sopra, Pd) concessi da Almerico II e sua moglie Franca al monastero di San Michele Arcangelo di Brondolo, situato a sud di Chioggia (Ve), è infatti, menzionato un "fosso Sancti Mari", prossimo alla palude di Anguillara (B. LANFRANCHI STRINA, SS. Trinità e S. Michele Arcangelo di Brondolo. II. Documenti 8oo-1199, Fonti per la Storia di Venezia, Sez. II, Archivi ecclesiastici, Diocesi Clodiense, Venezia, n. 2). Ė plausibile che la chiesa, della quale non sussistono tracce nella documentazione successiva, si trovasse in loc. "Santa Maria della Torre al Grotaro", sul dosso che attraversava, in direzione NW, il centro di San Luca sino a loc. "La Grimana" a sud di Pozzonovo.

${ }^{27}$ Manca un preciso asse di simmetria, nonostante i diversi elementi di discontinuità presenti come il motivo a S affrontate e il cantaro stilizzato della seconda cornice o il grande giglio del registro centrale. Si noti, inoltre, la fattura piuttosto ingenua di alcuni fiori el'assenza del giglio angolare nella seconda cornice. ${ }^{28}$ La pieve di Santa Giustina di Monselice è attestata per la prima volta nel 968 quando l'arciprete Martino figura come acquirente di terreni in Montegaldella, nel comitato vicentino, in A. GLORIA (a cura di), Codice Diplomatico Padovano: dal secolo sesto a tutto l'undecimo, Venezia, 1877 (da ora CDP, I), n. 51. 
volume). Segnaliamo un pilastrino di pergula custodito presso il deposito comunale, di provenienza ignota ${ }^{29}$, ma ipoteticamente attribuito da G.P. Brogiolo all'arredo della chiesa altomedievale di San Pietro (fig. 10). La fronte, incorniciata da un listello liscio, è decorata da un doppio cordoncino formante un motivo a spinapesce che si sviluppa dai vertici inferiori, culminante con una foglia lanceolata profilata; ai lati si sviluppa una serie di foglie allungate a forma di palma, che trova un parallelo preciso in una lastra laterale dell'altare di Ratchis $(737-744)^{30}$, ma anche in alcuni pezzi bresciani di epoca liutprandea ${ }^{31}$. Il motivo a doppio cordoncino ritorto è riscontrabile in un dossale di cattedra in un pilastrino da Aquileia ${ }^{32}$, in tre frammenti di pluteo da Trento ${ }^{33}$, uno da Vicenza ${ }^{34}$, in un fronte di sarcofago
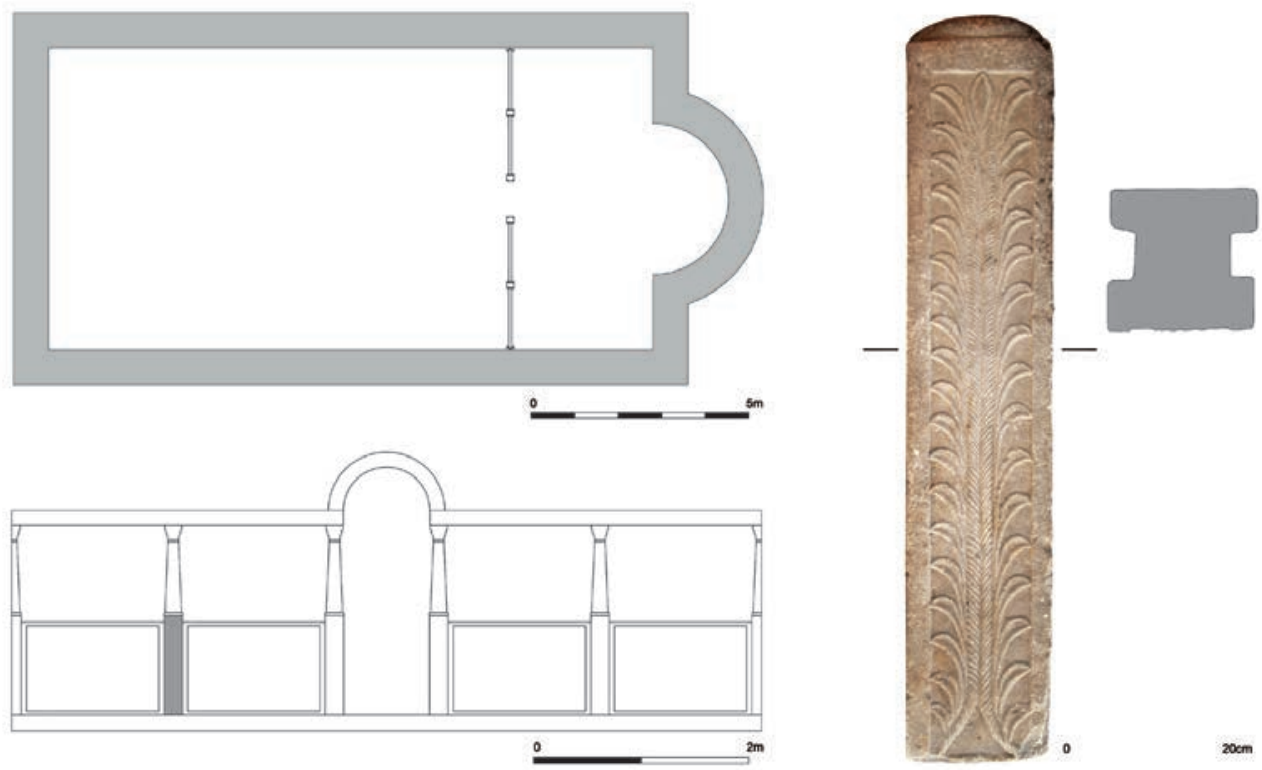
da Murano ${ }^{35} \mathrm{e}$ in vari manufatti di ambito cividalese ${ }^{36}$, tutti databili tra la fine dell'VIII e i primi decenni del IX secolo.

Dal mercato antiquario, forse veneziano, potrebbe essere giunto un frammento di arco inglobato nel muro di contenimento in laterizi a est della cappella del Castello Cini (fig. $11)^{37}$. Il pezzo è riconosciuto dalla critica come databile al pieno IX secolo ${ }^{38}$, ma un esame attento consente di sollevare alcuni elementi di dubbio sulla sua autenticità, a partire dallo schema compositivo totalmente insolito nel panorama della scultura altomedievale: l'archivolto è ornato da una larga modanatura liscia mentre una cornice a tre listelli delimita lo spazio triangolare interno occupato da un pavone, fiori a sei petali, un giglio e una croce mediana ad estremità patenti. Il principale indizio di modernità si ravvisa, tuttavia, in alcuni segni di lavorazione peculiari di una strumentazione meccanica, come la fitta puntinatura sulla superficie di fondo o l'intaglio regolare di alcuni particolari (ad esempio il solco centrale dell'arco e il profilo del volatile) o ancora nella finta rottura di sinistra che "risparmia" il fiore. La scultura

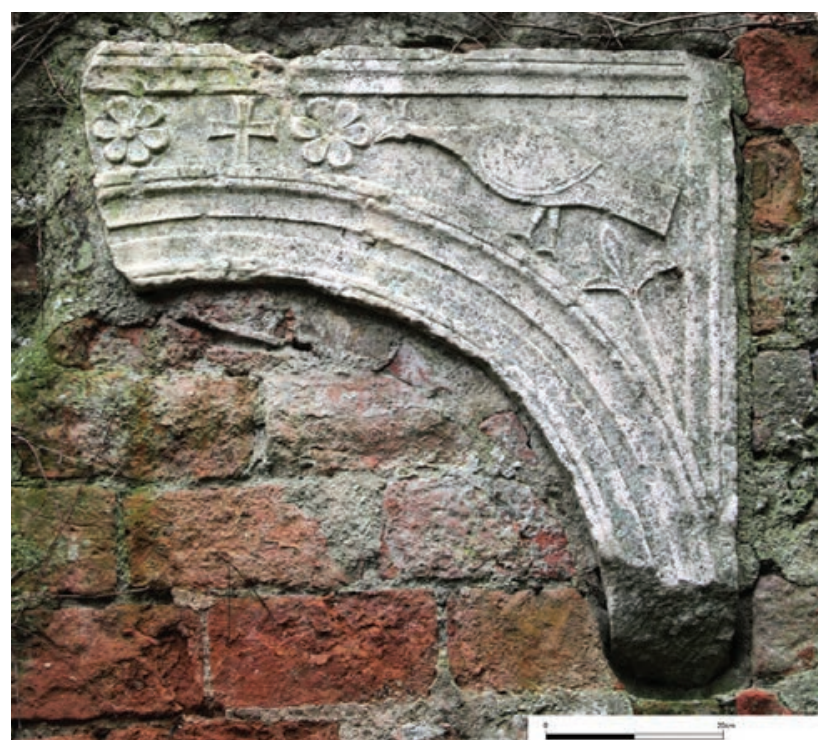

Fig. 11. Monselice (Pd), Castello Cini, frammento di arco.

${ }_{29}$ Dimensioni: cm 17,5 x 84 X 16,5. Sulle facce laterali si riconoscono gli incassi a sezione trapezoidale per la messa in opera dei plutei. Il manufatto conserva la base con foro centrale della colonnina in monoblocco che doveva esservi sovrapposta (raggio $\mathrm{cm} \mathrm{16).} \mathrm{Il} \mathrm{pezzo} \mathrm{è} \mathrm{riprodotto} \mathrm{per} \mathrm{la} \mathrm{prima} \mathrm{volta} \mathrm{dal} \mathrm{Cattaneo,}$ che si limita a darne una sommaria descrizione (R. CATTANEO, L'architettura in Italia: dal secolo 6 al Mille circa, Venezia, 1889, p. 106).

$3^{30}$ Lastra laterale sinistra (Visitazione), cfr. A. TAGLIAFERRI, Corpus della scultura altomedievale. X. Le diocesi di Aquileia e Grado, Spoleto (PG), $1981, \mathrm{n}$. 312. ${ }^{31}$ Un pluteo riutilizzato nella Rotonda romanica di Santa Maria Assunta (G. PANAZZA, A. TAGLIAFERRI, Corpus della scultura altomedievale. III. La diocesi di Brescia, Spoleto (PG), 1966, n. 25); una lastra con croce gemmata di San Savatore (G. PANAZZA, A. TAGLIAFERRI, Corpus... n. 62), datati alla metà dell'VIII secolo, un pluteo da Santa Maria Assunta di Gussago in Franciacorta (G. PANAZZA, A. TAGLIAFERRI, Corpus... n. 201).

${ }^{32}$ G. PANAZZA, A. TAGLIAFERRI, Corpus... nn. 273-293.

33 Visibile su un pluteo da San Vigilio, valido come confronto anche per il motivo a palma, datato all'epoca dei lavori del vescovo Iltigario, all'inizio dell'IX secolo (P. PORTA, Per il corpus..., p. 47); su due frammenti da Santa Maria Maggiore, inquadrati tra l'ultimo quarto dell'VIII secolo e il primo quarto del IX (M. BEGHELLI, Scultura altomedievale dagli scavi di Santa Maria Maggiore a Trento, Bologna, 2013, invv. 2657, 3213).

${ }^{34}$ Nella chiesa dei Santi Felice e Fortunato, assegnata prudentemente alla cosiddetta maestranza berico-benacense (E. NAPIONE, Corpus della scultura altomedievale. XIV. La diocesi di Vicenza, Spoleto (PG), 2001, n. 79).

35 M. VECCHI, Sculture tardo-antiche..., n. 123.

${ }^{36}$ Scolpito sul retro del cosiddetto pluteo di Paolino, datato al terzo quarto dell'VIII sec. (A. TAGLIAFERRI, Corpus..., n. 333), in un frammento di dossale del Museo Nazionale di Cividale della metà dell'VIII sec. (A. TAGLIAFERRI, Corpus... n. 401), in un pluteo da Santa Maria Assunta (A. TAGLIAFERRI, Corpus... n. 334) ma anche, rimanendo in ambito friulano, in un frammento di pluteo di VIII sec. da San Martino a Rive d'Arcano (S. LUSUARDI SIENA, L'arredo liturgico..., p. 172, n. 12) e in una cornice da Nimis (Ud) (A. TAGLIAFERRI, Corpus... n. 473).

37 Il pezzo figura nell'inventario dattiloscritto delle collezioni del castello di Monselice (a. 1950, n. 6106) con la seguente descrizione: "Arte bizantina. Arco frammentario di una porta con una croce, pavoni e fiori". Sono omesse le voci riguardanti la provenienza e la data di acquisto (Fondazione Giorgio Cini, Archivio dell'Istituto di Storia dell'Arte). Dimensioni: $\mathrm{cm} 78,9$ x 80.

${ }^{38}$ Per primo Roberto Valandro che, sulla base di testimonianze orali, ne ipotizza la provenienza dalla vicina località di Pernumia (R. VALANDRO, Appunti per la conoscenza della Iudiciaria longobarda di Monselice nella documentazione storica e archeologica, in Atti e memorie del Sodalizio Vangadiciense, II, 1982, 

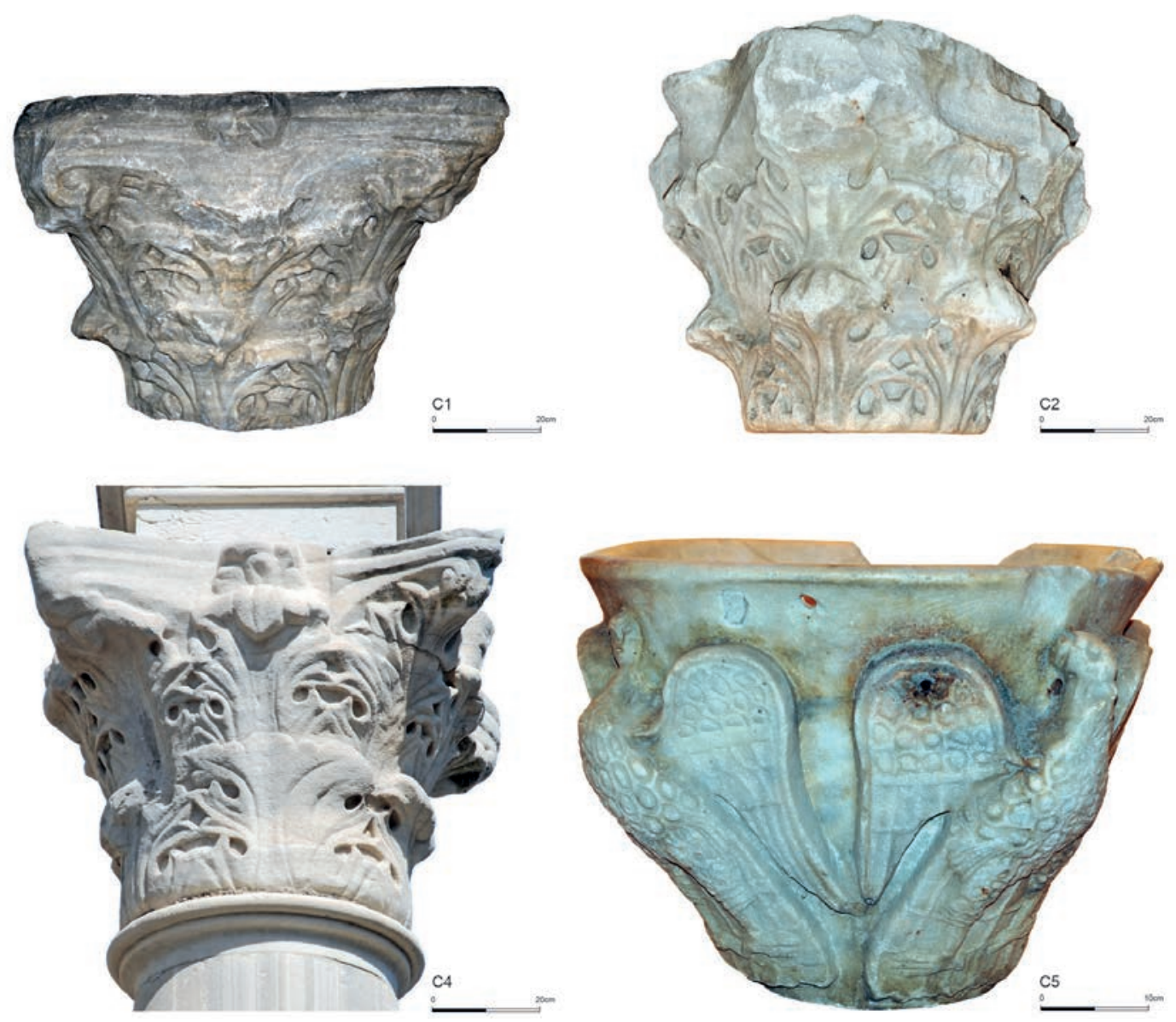

Fig. 12. Chioggia (Ve), capitelli bizantini.

rientrerebbe, quindi, a pieno titolo negli elementi di arredo architettonico acquistati nel corso degli anni ' 30 da Vittorio Cini, su consiglio del critico d'arte Eugenio Barbantini, per enfatizzare l'aspetto medievale del castello.

Il nostro breve itinerario termina a Chioggia (Ve), dove i manufatti si fanno più consistenti e permettono di evidenziare un quadro produttivo piuttosto unitario. I più importanti repertori iconografici sulla città lagunare e i suoi monumenti antichi sono senza dubbio le "Antichità di Chioggia sacre e profane", illustrate nel 1763 dall'olandese Giovanni Grevembroch su commissione del vescovo Giannagostino Gradenigo 39 e la serie di disegni prodotti agli inizi del '90o da Aristide Naccari e conservati, per la maggior parte, nell'archivio diocesano della città ${ }^{0}$. La formazione romantica dell'artista clodiense lo portò ad interessarsi particolarmente ai manufatti e alle architetture di epoca medievale; in questa prospettiva l'individuazione e la documentazione grafica di oggetti e architetture, che indirettamente costituiscono lo stato delle evidenze artistiche (e archeologiche) visibili fino ad allora in città, divenne un obiettivo seguito con accuratezza e dedizione. Oggetto di interesse sono le tavole ad acquerello eseguite nel 1904, oggi raggruppate nel Fondo
A. Naccari, fasc. 12, Antichità di Chioggia conservato presso l'Archivio Diocesano clodiense. Fra queste, quattro raffigurano un gruppo eterogeneo di manufatti bizantini (4 capitelli e 4 fusti di colonna), corredati da descrizioni, dimensioni e informazioni didascaliche sul contesto di ritrovamento. La restituzione particolareggiata dei reperti ne ha reso semplice l'individuazione fra alcuni pezzi dislocati oggi nella città: si tratta di un capitello reimpiegato in tempi recenti come supporto di vasca battesimale nella chiesa di San Giovanni Battista (C.1 nel catalogo in appendice); uno conservato nel Museo Civico della Laguna Sud (C.2); un altro custodito nel Museo Diocesano (C.3); un quarto capitello messo in opera su un fusto di epoca romana al centro di piazza Vigo (C.4) (fig. 12); tre fusti di colonna reimpiegati in una fontana posta a ridosso del perimetrale sud della cattedrale (F.1-3; fig. 13). A questi dobbiamo aggiungere un capitello figurato di epoca mediobizantina, conservato presso il Museo Diocesano, l'unico tra gli esemplari elencati illustrato da Grevembroch quando ancora era in uso come contenitore per le offerte nella chiesa di San Domenico ${ }^{4}$.

Tralasciando brevi pubblicazioni di carattere locale ${ }^{42}$, a questo gruppo di manufatti, che possiamo includere nel

p. 106); Annalisa Colecchia, invece, lo pone in relazione con la chiesa altomedievale di San Tommaso (A. COLECCHIA, Scheda pd.56 in G. P. BROGIOLO, M. IBSEN (a cura di), Corpus Architecturae Religiosae Europeae (saec. IV-X), v. II, 1. Province di Belluno, Treviso, Padova, Vicenza, Zagreb, 2009, p. 203).

39 Antichità di Chioggia sacre e profane scelte da Giannagostino Gradenigo e disegnate da Giovanni Grevembroch nel 1763; illustrate da Girolamo Ravagnan nel 1835, Chioggia, 1996.

${ }^{40}$ ADC, Fondo A. Naccari, fasc. Antichità di Chioggia.

${ }^{41}$ Antichità di Chioggia..., tav. XXIX.

${ }^{42}$ G. MARANGON, Frammenti di mistero, Chioggia, 2001, pp. 38-39; G. MARANGON, Marmi e ceramiche del museo diocesano di Chioggia, Chioggia, 2011, p. 59. I pezzi non sono presi in considerazione del lavoro di Rizzi dedicato alla scultura erratica di Chioggia (A. RIZZI, Scultura erratica a Chioggia, in Antichità viva, XII, 3, pp. 13-26). 
corpus delle spoglie marmoree bizantine in area lagunare e nell'entroterra veneziano ${ }^{43}$, non è stato ad oggi dedicato uno studio specifico. In questa sede porremo, quindi, l'attenzione sull'aspetto tipologico e stilistico dei pezzi, proponendone una prima classificazione in appendice.

La questione della provenienza dei manufatti individuati e del momento in cui arrivarono a Chioggia deve necessariamente rimanere aperta. Tutti gli esemplari, infatti, sono erratici e per alcuni di essi non abbiamo indizi che ci consentano di risalire a periodi anteriori alla fine del XVIII secolo. La maggior parte dei materiali è inquadrabile cronologicamente tra la seconda metà del V e la metà del VI secolo e classificabile come importazione dall'area costantinopolitana. Tre dei quattro capitelli mostrano caratteristiche uniformi, sia per stile che per dimensioni. I tre fusti sono del tutto analoghi tra loro, salvo qualche piccola differenza nellealtezze ${ }^{44}$, tanto da far supporre la loro messa in opera in un medesimo edificio. In questo senso, è solo a livello di ipotesi che possiamo collegarli con la cattedrale medievale di Santa Maria (distrutta da un incendio nel 1623), del cui aspetto architettonico e decorativo si ha testimonianza nelle narrazioni
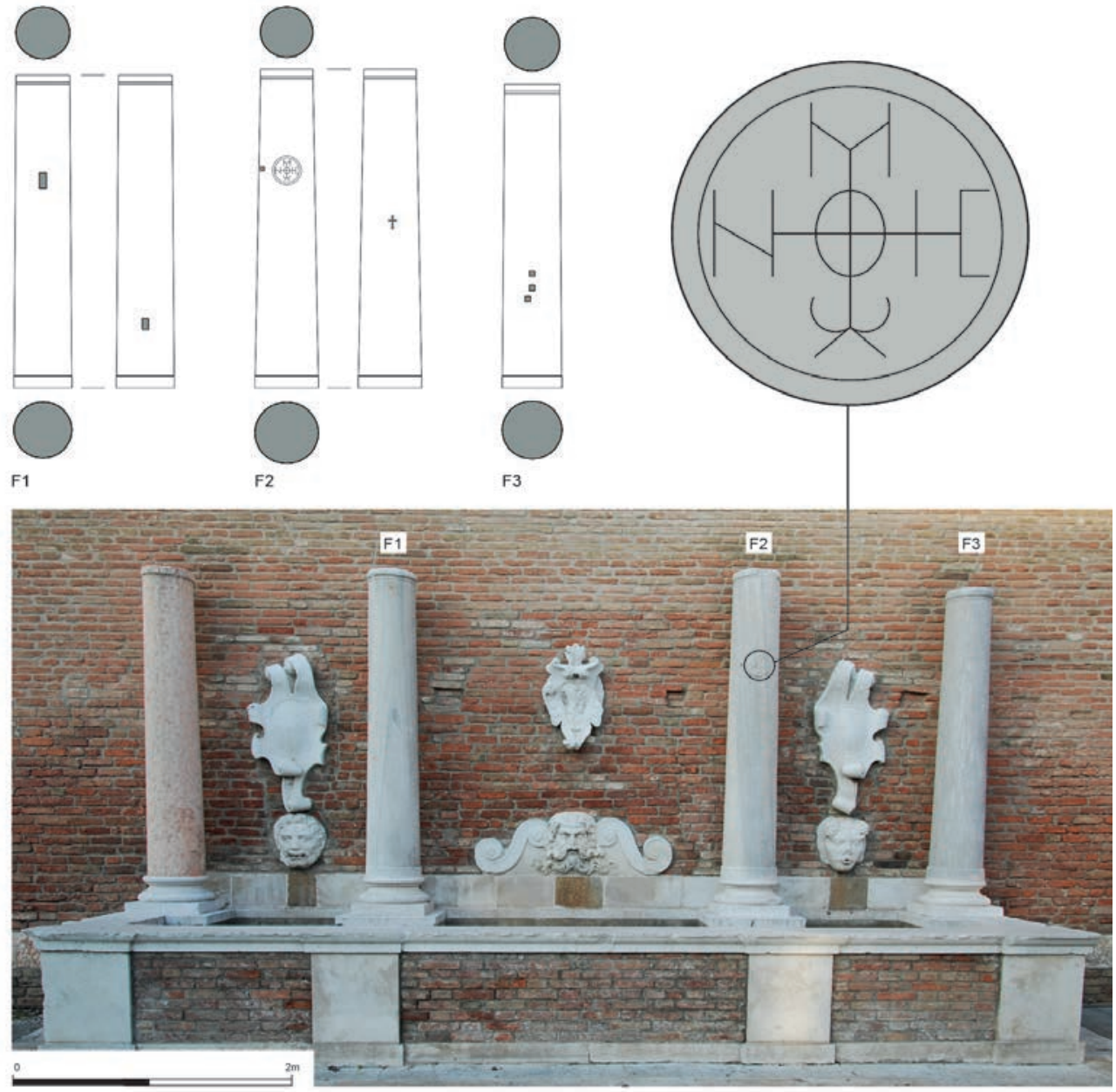

Fig. 13. Chioggia (Ve), colonne di piazza Perottolo (restituzione grafica).

dello storico Pietro Morari (1569) ${ }^{45}$ e nella visita pastorale del vescovo Pasquale Grassi $(1619)^{46}$. Gli autori descrivono scrupolosamentel'interno dell'edificio, mettendone in risalto soprattutto l'arredo architettonico e la consistente presenza di colonne in marmo bianco "finissimo", almeno in parte di

derivazione classica: due ordini di nove colonne dividevano le navate ${ }^{47}$; dieci colonne inframezzate da plutei andavano a formare l'iconostasi, sormontata da croce dorata e da sedici grandi statue ${ }^{48}$; ai lati due amboni "sostenuti da sedici, o venti colonne parte serpentine, parte di pario, et altri marmi singu-

${ }_{43}$ Consistenti le attestazioni di reimpiego di materiale scultoreo di provenienza costantinopolitana, soprattutto a Venezia: è il caso dei materiali utilizzati per l'arredo esterno della basilica marciana (I. FAVARETTO et alii (a cura di), Marmi della basilica di San Marco: capitelli, plutei, rivestimenti, arredi, Milano, 2000 con bibliografia precedente), dei capitelli reimpiegati in alcuni edifici residenziali di XIII secolo (C. BARSANTI, Venezia e Costantinopoli: capitelli di reimpiego nelle dimore lagunari del Duecento, in Hadriatica. Attorno a Venezia e al Medioevo tra arti, storia e storiografia. Scritti in onore di Wladimiro Dorigo, Padova, 2002, pp. 59-69), dei capitelli tardoantichi della chiesa di San Giacomo di Rialto (C. BARSANTI, L'esportazione di marmi dal Proconneso nelle regioni pontiche durante il IV-VI secolo, in RIA, n.s. III, 12, 1989 (1990), p. 135, n. 180), del gruppo di sei capitelli a imposta reimpiegati nella chiesa dei Santi Giovanni e Paolo (C. BARSANTI, M. PILUTTI NAMER, Da Costantinopoli a Venezia: nuove spoglie del San Polieucto. Nota preliminare, in Nea Rhome, 6, 2010, pp. 133-156), degli otto capitelli di età paleobizantina reimpiegati nelle edicole dei Frari (M. PILUTTI NAMER, Su alcuni spolia bizantini d'eccezione: $i$ capitelli di navata delle edicole dei Frari, in Venezia Arti, 22/23, 2008/2009, pp. 2-10), dei frammenti di lastre reimpiegati nel monastero di San Giorgio Maggiore (R. FLAMINIO, Frammenti di sculture bizantine nel monastero di San Giorgio Maggiore a Venezia, in Venezia Arti, 12, 1998, pp. 5-16). Sui capitelli della cattedrale di Torcello e della vicina chiesa di Santa Fosca, si veda A. GUIGLIA GUIDOBALDI, Reimpiego di marmi bizantini a Torcello, in A. IACOBINI, E. ZANINI (a cura di), Arte profana e arte sacra a Bisanzio, Roma, 1995, pp. 603-632; per gli esemplari di San Donato di Murano si veda L. SPERTI, Originali tardoantichi e protobizantini e imitazioni medioevali tra i capitelli della chiesa di San Donato a Murano, in Società e cultura in età tardoantica. Atti dell'incontro di studi, Udine, 2004, pp. 229-252). Per l'entroterra veneziano basti ricordare: i plutei di Codevigo (C. BARSANTI, I plutei degli intercolunni delle gallerie, in A. GUIGLIA GUIDOBALDI, C. BARSANTI (a cura di), Santa Sofia di Costantinopoli. L'arredo marmoreo della Grande Chiesa giustinianea, in Studi di Antichità Cristiana, 6o, Città del Vaticano, 2004, p. 471), i capitelli del palazzo del Consiglio di Padova (C. BARSANTI, I "Catini d'oro" di Padova: spoglie costantinopolitane del VI secolo, in G. T. TROVABENE (a cura di), Florilegium artium. Scritti in memoria di Renato Polacco, Padova, Padova, pp. 37-48), i capitelli reimpiegati nel portico di casa Muschietti a Portogruaro (L. KRAMER, Skulpturen mit Adlerfiguren an Bauten des 5. Jahrhunderts n.Chr. in Konstantinopel, Köln, 1968, cat. n. 15, p. 91), gli archetti di ciborio di Santa Maria di Portogruaro (M. BONFIOLI, Tre arcate marmoree protobizantine a Lison di Portograuro, in Ricuperi bizantini in Italia, 1, 1979).

${ }_{44}^{44}$ La differenza doveva essere compensata reimpiegando basi o capitelli di altezze diverse.

${ }^{45}$ P. MORARI, Storia di Chioggia, Chioggia, 1870, pp. 25-31. Era un grande edificio di tipo basilicale, a tre navate chiuso a est da un'abside poligonale. Le attente descrizioni di Pietro Morari hanno rappresentato per Aristide Naccari un fondamentale punto di riferimento per varie ipotesi restitutive grafiche, oggi conservate presso l'Archivio Diocesano di Chioggia.

${ }^{46} \mathrm{ADC}$, vol. 55, c. 2v-4r.

${ }^{47} \mathrm{ADC}$, vol. 55, c. 2 v, 25 luglio 1619.

48 "...dalla tribuna alla terza colonna c'era un ordine di colonne bianche di finissimo marmo che attraversavano la chiesa..." (P. MORARI, Storia..., p. 26). 
lari"; quattro colonne sorreggevano, infine, la cupola mosaicata del ciborio ${ }^{49}$. Sostenere che le colonne (ma anche i capitelli) in esame appartenessero a questo complesso architettonico rimane per il momento solamente una teoria inverificabile, in mancanza del supporto dei dati archeologici, sullo sfondo di un contesto storico di cui quasi nulla conosciamo, almeno fino al XII secolo ${ }^{50}$. Allo stesso tempo l'ipotesi, anch'essa verosimile, di identificarli con alcune delle numerose spoglie marmoree costantinopolitane approdate in laguna nel corso del XII secolo consiglia di lasciare aperto il problema.

\section{CÁTALOGo}

\section{C.1. Chioggia, Chiesa di San Giovanni Battista, capitello corinzio \\ Collocazione attuale: Chioggia, chiesa di San Giovanni Battista (dal 1992), presbiterio ${ }^{51}$.}

Collocazione originaria: ignota.

Materiale: marmo proconnesio.

Stato di conservazione: buono. Si notano varie sbrecciature nelle volute.

Dimensioni: $\mathrm{cm} 45$ (diametro inferiore) x 53 (altezza).

Sigle: ЕП leggermente ruotata in senso orario tra una voluta e il piano spiovente sotto alla bugna d'abaco (sigla di atelier).

Descrizione: capitello corinzio, di taglia superiore alla media, riconducibile al tipo VII della classificazione del Kautzsch (cosiddetto "Lederblätter"), ampiamente esportato dall'area costantinopolitana nel Mediterraneo orientale specialmente nelle regioni interessate dalla politica edilizia giustinianea $^{52}$. Contraddistinto dall'assenza delle elici, mostra una struttura compatta, caratterizzata da due corone di acanto finemente dentellato, la superiore costituita da otto foglie, l'inferiore da sei. I confronti per questo tipo di capitello sono numerosi, basta ricordare il noto gruppo dei capitelli recuperati dal relitto naufragato a Marzamemi53. Cronologia: fine V, metà del VI secolo.

\section{C.2. Chioggia, Museo Civico della Laguna Sud, capitello corinzio \\ Collocazione attuale: Chioggia, Museo Civico della Laguna} Sud (dal 1997), già nel chiostro del convento dei Filippini fin dal $1992^{54}$.
Collocazione originaria: ignota.

Materiale: marmo proconnesio.

Stato di conservazione: discreto. Si notano diffuse fratture nella parte superiore.

Dimensioni: $\mathrm{cm} 45$ (diametro inferiore) x 54 (altezza).

Sigle: EP speculare, tra una voluta e il piano spiovente al di sotto della bugna d'abaco. Il Naccari disegna un'altra sigla di atelier (EY), posta in posizione simmetrica alla precedente e oggi scomparsa a causa del degrado del manufatto.

Descrizione: il capitello è simile per dimensioni e tipologia a C.1, cui si rimanda per la descrizione.

Cronologia: fine V, metà del VI secolo.

\section{C3. Chioggia, Museo Diocesano, capitello corinzio (fig. 14)}

Collocazione attuale: Chioggia, Museo Diocesano.

Collocazione precedente: palazzo episcopale, portico del cortile meridionale (lato nord) ${ }^{55}$.

Materiale: marmo proconnesio.

Stato di conservazione: buono.

Dimensioni: cm 35 (diametro inferiore) x 42 (altezza).

Sigle: MA B. Una foglia trilobata rovescia, inserita nello spazio ridotto sotto la bugna d'abaco, divide la lettera aggiuntiva $\mathrm{B}$ dalle altre due ${ }^{56}$ (sigla di atelier).

Descrizione: capitello corinzio cosiddetto a "V" per la forma delle volute (il tipo 5 della classificazione del Kautzsch)57. La struttura decorativa consta di due corone di foglie di acanto spinoso a grandi dentelli, 4 nella superiore e 5 nell'inferiore. L'abaco ha i lati leggermente introflessi ed è bipartito mediante una profilatura a listelli Lo spazio ridotto sotto la bugna d'abaco accoglie una foglia trilobata rovescia con nervatura centrale. Sul piano di attesa superiore reca due incavi quadrangolari di grappa (larghezza $\mathrm{cm} \mathrm{8}$, profondità $\mathrm{cm} \mathrm{3,5),} \mathrm{uno} \mathrm{dei} \mathrm{quali} \mathrm{conserva} \mathrm{ancora}$ residui di piombo. Il manufatto rientra nella produzione microasiatica riconducibile alle botteghe delle cave del Proconneso. Tra i confronti per questo tipo di capitello ricordiamo un esemplare di area pontica nel Museo di Costanza e di Burgas in Bulgaria, simile a un pezzo del Museo Archeologico di Istanbul (inv. 1228) s. $^{8}$.

Cronologia: seconda metà del V secolo, metà del VI secolo.

\footnotetext{
49 "...Hoc altare quatuor gradus marmoreos, supra cupulam marmoream pulchram habentem coelum musaici figuratum, quatuor columnis marmoreis magni valoris suspensam..." (ADC, vol. 55, cc. 4 r-v, 26 luglio 1619).

${ }^{50}$ La prima menzione della pieve di Santa Maria di Chioggia è riportata da Girolamo Vianelli: si tratterebbe di un atto pubblico datato 1091, oggi perduto, nel quale è menzionato un "Johannes prēsbr Plebanus Sancte Marie gemino comune" (G. VIANELLI 179o, Nuova serie..., p. 294, n. IV). La chiesa fu elevata al titolo di cattedrale dopo il 1110, anno della fondazione giuridica della diocesi clodiense, con la traslazione della decaduta sede vescovile di Malamocco (M. DE BIASI, L'antica Metamauco sede vescovile, in Ateneo Veneto, 177, 1990, pp. 305-314).

${ }^{51}$ Ai tempi del Naccari era custodito nel deposito comunale presso il mercato orticolo di Brondolo (ADC, Fondo A. Naccari, fasc. Antichità di Chioggia, dis. 3). ${ }^{52}$ R. KAUTZSCH, Kapitellstudien: Beitrage zu einer Geschichte des spatantiken Kapitells im Osten vom Vierten bis Siebente Jahrhundert, Berlin, 1936, p. 61. Sul tipo e la diffusione si veda C. BARSANTI, L'esportazione di marmi..., pp. 111-125.

53 Vd. G. KAPITÄN, Elementi architettonici per una basilica dal relitto navale del VI secolo di Marzamemi (Siracusa), in Corsi di Cultura sull'Arte Ravennate e Bizantina, 27 (1980), p. 81 ss., fig. 6.

${ }^{54}$ In precedenza e già ai tempi del Naccari il pezzo era sistemato nel deposito comunale presso il mercato ortofrutticolo di Brondolo. Presenta un numero di inventario in corrispondenza del piano di appoggio inferiore (RA81), ma rimane ignota la circostanza di siglatura.

${ }^{55}$ Lo deduciamo dal disegno n. 6 del Naccari, che raffigura il capitello come appariva nel momento della scoperta durante alcuni scavi eseguiti in data 19 luglio 1894. La colonna faceva probabilmente parte di un portico esterno, costruito nel 1474 dal vescovo Nicolò Traversi (G. VIANELLI, Nuova serie de' vescovi di Malamocco e di Chioggia accresciuta e con documenti in gran parte ora sol pubblicati, Venezia, 1790, p. 65), del quale rimane ancora traccia nelle due colonne in granito poste ai lati dell'ingresso della "sala Piasentini".

${ }^{56}$ Un'analoga sigla decora alcuni capitelli corinzi, di tipo Kautzsch VIII, reimpiegati all'interno della cisterna di Yerebatansaray di Istanbul (segnalazione di Giulia Marsili, che desidero ringraziare per gli utili consigli).

${ }^{57}$ R. KAUTZSCH, Kapitellstudien..., p. 56 ss. Sul tipo e la diffusione si veda C. BARSANTI, L'esportazione di marmi..., pp. 125-135, A. GUIGLIA GUIDOBALDI, Scultura bizantina in Lombardia: i capitelli di Leggiuno, in Arte d'Occidente, temi e metodi. Studi in onore di Angiola Maria Romanini, Roma, 1999, p. 290.

${ }^{58}$ C. BARSANTI, L'esportazione di marmi..., p. 128, fig. 39; R. KAUTZSCH, Kapitellstudien..., p. 59, n. 184.
} 

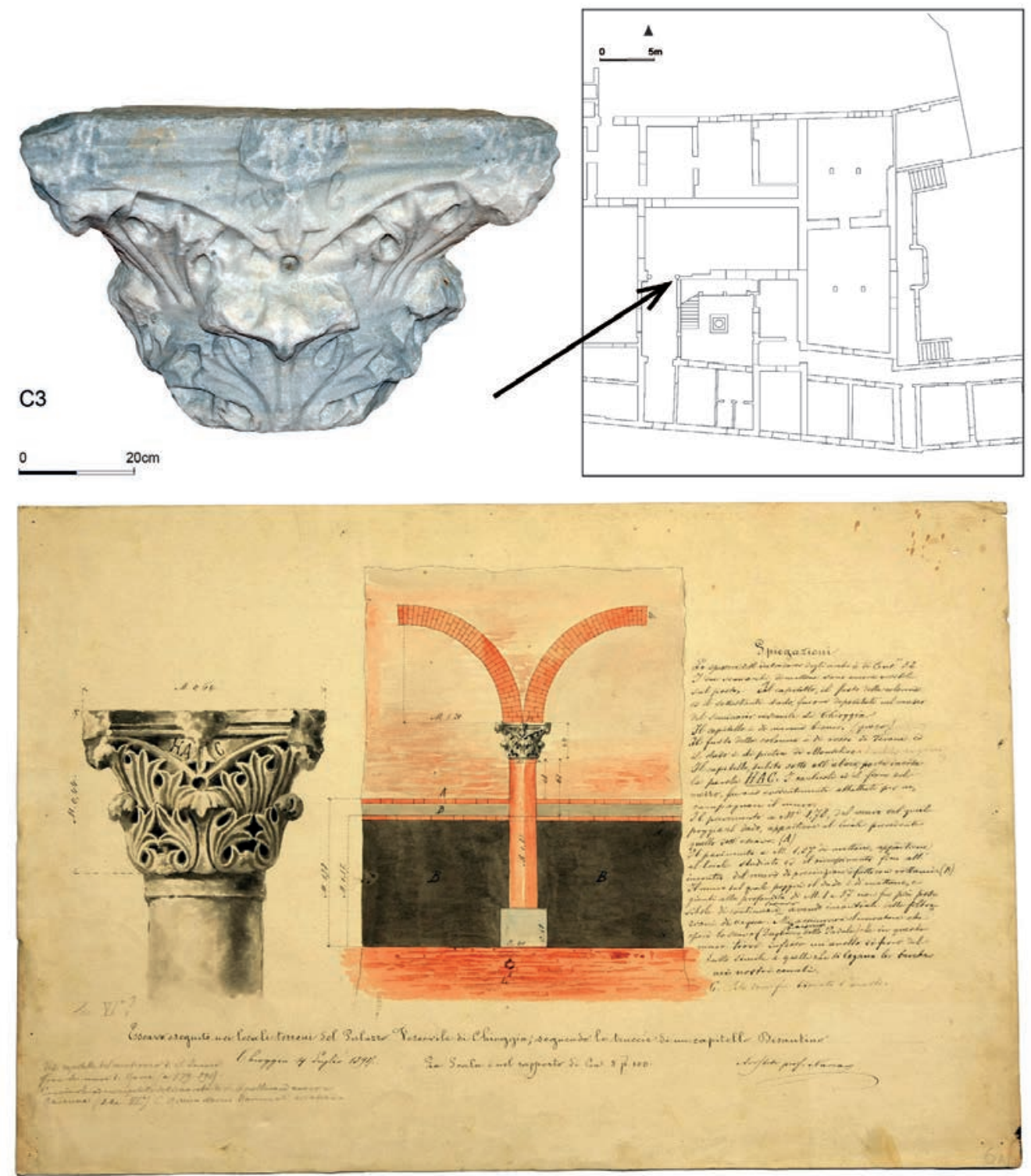

Fig. 14. ADC, Fondo A. Naccari, fasc. Antichità di Chioggia, dis. 1 (per gentile concessione dell'Archivio Diocesano di Chioggia, autorizzazione 21/12/2015).

C4. Chioggia, piazza Vigo, capitello corinzio

Collocazione attuale: piazza Vigo (dal 1786)59.

Collocazione originaria: ignota.

Materiale: marmo proconnesio.

Stato di conservazione: buono. Si rileva un tassello di restauro in corrispondenza della corona inferiore.

Dimensioni: cm 40 (diametro inferiore) x 46,3 ca. (altezza).

Sigle: assenti.

Descrizione: capitello corinzio riconducibile al tipo VII della classificazione del Kautzsch. L'abaco ha i lati introflessi ed è suddiviso mediante una profilatura a tre listelli. Elemento distintivo di questo esemplare è la presenza di una croce latina a bracci patenti su una bugna semicilindrica dell'abaco.

Cronologia: fine V, metà del VI secolo.

\section{C5. Chioggia, Museo Diocesano, capitello figurato}

Collocazione attuale: Chioggia, Museo Diocesano (dal $2002)^{60}$.

Collocazione originaria: ignota.

Materiale: marmo proconnesio (?).

Stato di conservazione: discreto. Il capitello è stato scavato al suo interno per una profondità di ca. cm 21,5. Presenta, al di sotto del margine superiore, tre incavi quadrango-

59 Il fusto, che sorregge in modo non congruo il capitello, fu eretto nel 1786 durante i lavori di risistemazione della piazza voluti dal podestà Giulio Antonio Mussati. Venne recuperato nel 1763 durante i lavori di ricostruzione dell'antica torre meridionale del palazzo comunale assieme ad altri due esemplari, collocati nell'atrio dell'allora Cancelleria Civile e oggi dispersi (C. BULLO, Gli ultimi podestà della Repubblica Veneto in Chioggia, continuazione della serie di G. A. Gradenigo, Venezia, 1876, p. 18), Lo testimoniano le iscrizioni incise alla base del monumento (IVLIO ANTONIO MVSSATI PRAETORE OPT. MERITO/CIVITAS EREXIT A.D CIDIJCCLXXXVI). È alto m 3,48 con un diametro inferiore di $\mathrm{cm}$ 47,75. All'estremità superiore una fessura trapezoidale pare indicare un tassello di restauro che avrebbe integrato una piccola parte del sommoscapo. Un tassello di restauro è presente anche sul lato opposto. La base in calcare e il collarino in marmo che la divide dal capitello sono moderni. Possiamo riferirlo all'età romana imperiale.

${ }^{60}$ Già nella chiesa di San Domenico ai tempi del vescovo Gradenigo (1763), reimpiegato come contenitore per le offerte e, prima ancora, presso il monastero di San Francesco fuori le mura, utilizzato come acquasantiera (Antichità di Chioggia, pp. 25-26, tav. XXIX).

${ }^{61}$ Questo foro presente nella conca potrebbe essere indizio di un precedente riutilizzo del manufatto come bacino per le acque di purificazione delle cose benedette (cfr. le voci "Lavage liturgique" e "Lavement" in DACL, v. 8/2, pp. 2001-2009). 
lari contigui e un foro da un lato, due fori quadrangolari dall'altro e un foro in corrispondenza del fondo ${ }^{61}$.

Dimensioni: cm 24,5 (diametro inferiore) x 39 (altezza). Sigle: assenti.

Descrizione: capitello figurato composto da quattro aquile ad ali distese, il cui piumaggio è reso in maniera astratta; disposte lungo tutta l'altezza del kalathos da cui sporgono per ca. cm 2,5, dovevano originariamente reggere gli angoli dell'abaco, oggi scomparso. Lo spazio tra un animale e l'altro si presenta privo di decorazione e ben lisciato, probabilmente in occasione della sua rilavorazione. Due aquile mostrano incisi, in corrispondenza della teste, due volti dai tratti somatici estremamente semplificati. Il capitello è tipologicamente confrontabile con un esemplare del museo di Iznik (inv. 670 ${ }^{62}$; per quanto riguarda, invece, la resa del piumaggio, due confronti sono offerti da un capitello custodito nella Dundarbey Medrese di Egridir e da uno nel Museo Archeologico di Istanbul (inv. 4722). Cronologia: X-XI secolo.

\section{F1-3. Chioggia, piazza Perottolo, fusti di colonna}

Collocazione attuale: Chioggia, piazza Perottolo, fontana adiacente al lato sud della cattedrale, (già nel cortile della Congregazione di Carità presso l'antica chiesa di Sant'Agnese nel 1893, quando lo storico Vincenzo Bellemo li descrive ${ }^{63}$ ).
Collocazione originaria: ignota.

Materiale: marmo proconnesio.

Stato di conservazione: buono.

Dimensioni:

F1: cm 42,2 (diametro inferiore); 39 (diametro superiore); m 2,35 (altezza).

F2: cm 46 (diametro inferiore); 38 (diametro superiore); $\mathrm{m}$ 2,39 (altezza).

F3: $\mathrm{cm} \mathrm{43,3} \mathrm{(diametro} \mathrm{inferiore);} 40$ (diametro superiore); m 2,28 (altezza).

Descrizione: fusti dei quali si conserva il sommoscapo con tondino a fascia e listello sottostante e l'imoscapo a fascia alta cm 9. Non si percepisce l'entasi. Le basi sono moderne. I manufatti presentano, a diverse altezze, svariati fori di incasso rettangolari evidentemente per l'inserimento di transenne ${ }^{64}$

Sigle: il fusto $\mathrm{F}_{2}$ presenta di un monogramma cruciforme a rilievo (diametro $\mathrm{cm} 18$ ), scolpito a ca. $\mathrm{cm} 65$ dal sommoscapo $^{65}$. L'imoscapo di F3 presenta inciso il numerale greco $\prod$.

Cronologia: Fusti di questo tipo sono prodotti dall'età imperiale a quella tardoantica. La presenza del monogramma (sicuramente più tardo) consiglia di sospendere il giudizio circa la cronologia dei manufatti.

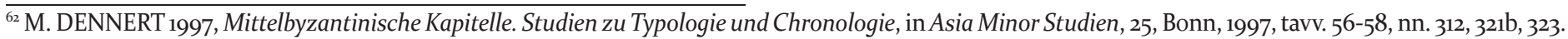
${ }_{63}$ V. BELLEMO, Il territorio di Chioggia, Chioggia, 1893, p. 57. Per due di essi l'autore ipotizza la provenienza da alcuni lavori di scavo del vicino canale Lombardo, avvenuti intorno alla metà del XIX secolo; tuttavia l'assenza di un evidente degrado superficiale non farebbe pensare a una loro prolungata persistenza in ambiente marino. Stando alla descrizione del Bellemo, il cortile custodiva, inoltre, due capitelli “senz'abaco...rivestiti di foglie di acanto", le cui dimensioni non sembrano trovare corrispondenza in quelle dei manufatti in esame ( $\mathrm{cm} 50$ di altezza per un diametro inferiore di $\mathrm{cm} 42$ ).

${ }^{64} \mathrm{~F}$ : presenta due incassi, il primo a un'altezza di $\mathrm{m} \mathrm{1,5}(\mathrm{cm} 5$ x 11,5 x 2,5), il secondo a un'altezza di $\mathrm{cm} 44(\mathrm{~cm} \mathrm{4,5} \times 8 \times 2,5)$. F2: nella metà superiore è

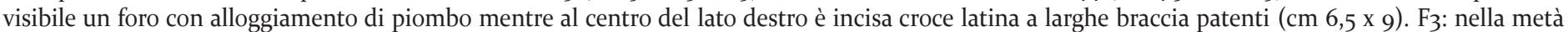
inferiore, a un'altezza rispettivamente di $\mathrm{cm} 84,73$ e 65 dall'imoscapo, sono presenti tre fori di incasso quadrangolari, due dei quali allineati ( $\mathrm{cm} 4 \mathrm{di}$ lato $\mathrm{x} \mathrm{cm}$ 3,5 di profondità).

${ }^{65}$ Non è stato possibile fino ad ora sciogliere il monogramma, formato dalle lettere greche I $\Lambda$ M N H C $\Phi \omega$.
} 